\title{
Research on the Influence Factors and Coordinated Control Strategies between Unit and Grid for Isolated Power System
}

\author{
Ge Jin ${ }^{1}$, Xiaomei Chen ${ }^{2}$, Yongxin Feng ${ }^{1}$, Shaoxiang Deng ${ }^{1}$, Hanting Yan ${ }^{2}$, Zexiang Cai ${ }^{2}$ \\ ${ }^{1}$ Electric Power Research Institute, Guangdong Power Grid, Guangzhou, China \\ ${ }^{2}$ School of Electric Power, South China University of Technology, Guangzhou, China \\ Email: mulanpurplelily@126.com
}

Received March, 2013

\begin{abstract}
As the existing coordinated control strategies between grid and unit have limitations in isolated power system, this paper introduces new coordinated control strategies which can improve the stability of isolated system operation. This paper analyzes the power grid side and unit side influence factors on the isolated power system. The dynamic models which are suitable for islanding operation are applied to simulate and analyze the stability and dynamic characteristics of the isolated power system under the conditions of different load disturbances and governor parameters. With considering the differences of frequency characteristics between the interconnected and isolated power system, the adjusting and optimization methods of under frequency load shedding are proposed to meet the frequency stability requirements simultaneously in the two cases. Not only proper control strategies of the power plant but the settings of their parameters are suggested to improve the operation stability of the isolated power system. To confirm the correctness and effectiveness of the method mentioned above, the isolated system operation test was conducted under the real power system condition, and the results show that the proposed coordinated control strategies can greatly improve stability of the isolated power system.
\end{abstract}

Keywords: Isolated Power System; Coordinated Control Strategies; Under Frequency Load Shedding; Dynamic Frequency Characteristics; Speed Governor Parameter Settings

\section{Introduction}

With the cross-regional interconnection of power system and the continuous expansion of power grid, the connections between systems at all levels are becoming closer and closer. And the single fault of power system, extreme weather or manual operation errors may possibly cause large area blackout accidents, and even cause the crash of power system. After that blackout, the power system is forced to take black start which is a self-help means after the power system accident and produces huge economic loss. However, when the large-scale power system fault occurs, we can take reservations and reasonable measures for the local power system, and keep isolated system operation stable. This can not only ensure the power supply for important load of the city, but also be helpful to restoration of the whole system which can reduce the economic loss.

In recent years, many countries around the world have occurred many largely influenced blackout accidents, such as the America and Canada blackout accident in 2003. In these accidents, a large number of regional grids which have the isolated system operation condition fail to make a full use of the coordinated control ability due to lack of effective coordinated control measures for the isolated system operation. And this leads to the unit tripping and the power lose of those areas [1-3].

There are some problems in research of possible risks grasp and control strategies for practical isolated power system operation. Firstly the characteristics and relevant coordinated control strategies of most power plants have not been considered for isolated system operation [4-7]. Then the stable operation of the power plant depends more on the major power system than the isolated power system as the effective power support.

With these problems, this paper analyzes the influence factors on the isolated system. Dynamic models which are suitable for the isolated power system are used to analyze the stability of frequency response under the different conditions such as load disturbances, speed governor parameters and logical control conditions. Optimization method of the under frequency load shedding is proposed to meet the frequency stability requirements 
in interconnected and isolated system operation with considering the differences of frequency characteristics in the two cases[8-10]. The parameter settings and control strategies are proposed to improve the isolated operational ability[11-16].

\section{Frequency Characteristics of Isolated Power System and Its Influence Factors}

\subsection{Frequency Characteristics}

For most heavy load in regional power system, the system response to the isolated network operation is basically a consistent frequency transient process which is often accompanied with voltage changing highly or lowly.

When the generated power is shortage in isolated network, the initial transient process is determined by the response of power plants spinning reserves and low-frequency low-voltage load shedding, often within seconds reaching the lowest frequency. After that point the system frequency response depends on the characteristics of prime mover and speed governor. With the surplus power in isolated system, the frequency will rise. Then the speed governor will work and give a response to reduce the mechanical power produced by the unit. The ability to maintain stability instead of losing load of the isolated network actually depends on the partly load shedding ability of the unit.

\subsection{Influences of the Power Grid}

The influence of power system primarily performances on the operation mode that is set. It is necessary for isolated power grid. So when the operation mode of the power system is made, all of the possible running situation that may occur in the isolated power system should be considered. The automation equipments of power system including the low-frequency low-voltage load shedding, and low frequency disconnection device as the last defense line of the power system are important in affecting the operation mode of the power system. With them, the collapse of frequency and voltage can be prevented in the accident and the partly power supply can be guaranteed. But if automation equipment such as the under frequency load shedding fails to take consideration on the frequency characteristics both of interconnected system and isolated system, it will cause frequency dropping dramatically during isolated network runtime, and the power system will collapse in the end.

\subsection{Influences of the Unit}

The steam turbine of each unit under different speed deviation has different cultivate times in total. It shows the lifetime of the unit. The low frequency operation of the steam turbine is more dangerous than the high one. Because after the frequency rises due to load shedding, it can be reduced by the governor or OPC, the overspeed protection control system, to regulate output power.

Figure 1 shows the lifetime of steam turbine under different frequency deviation. For power plant A, the two horizontal lines indicate that there is no loss and the turbine can run continuously between the frequency of 49.4 $\mathrm{Hz}$ and $50.6 \mathrm{~Hz}$. When the frequency drops to $46.5 \mathrm{~Hz}$, the turbine can only run for one second, as the lifetime curve shows at the time of frequency offset. The power plant B has a lower frequency than $50 \mathrm{~Hz}$, because of quickly action of its frequency governor. When the frequency is lower than $50 \mathrm{~Hz}$, the life time of the turbine will stepped reduce.

When isolated network is forming, some fixed values of the protection devices may affect the stability of isolated system operation, while current protection of the unit and its fixed values are setting mainly based on interconnected network operation. So it is necessary to set reasonable values for the generator protection, which will not only ensure safe operation of the unit, but also prevent the unstable operation or trip of the units. The following generator protection equipments should be considered during the isolated system operation: electrical overspeed trip, mechanical overspeed trip, turbine overspeed protection control (OPC), low and over voltage protection of generator, and auxiliary protection settings.

\section{Dynamic Models of Isolated Power System}

The influence of the unbalanced power on dynamic frequency characteristic of the power system at the moment of isolated system formation can be analyzed by the models in isolated network. Considering the unit in city grid mainly uses the turbine generator, especially the reheat type as shown in Figure 2. The domain time constant of this turbine model is the thermal time constant $T_{R}$. The constant $K_{m}$ is the total gain to realize the adjustment of output power [8].

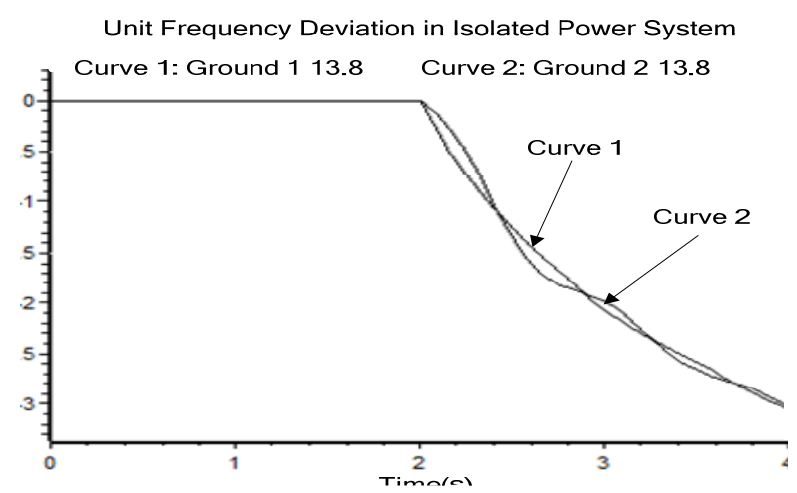

Figure 1. Turbine lifetime with different frequency offsets 
Typical speed governor model is shown in Figure 3.

The most important parameter of speed governor is the differential coefficient. It is marked with $\mathrm{R}$ in the feedback element of the above figure. And a simplified model of power system is established based on including its important dynamic characteristics. By integrating reheat steam turbine model with speed governor model we get the reduced order system frequency model, as shown in the Figure 4.

There are six constants to describe the characteristics of this model: gain coefficient $K_{m}$, damping coefficient D, inertia constant $H$, thermal time constant $T_{R}$, power proportion of high pressure cylinder $F_{H}$, and differential coefficient $\mathrm{R}$.

The incremental power setting of governor and output electromagnetic power of generator are the two input variables of the model. Governor incremental power value is adjusted according to the unit instruction. The output setting value needs to meet requirement of the load. As at the moment of isolated network forming what is corresponding to the disturbance are system characteristics of just few seconds, so the governor incremental power value can be considered to be constant. So electromagnetic power variation can be seen as a disturbance power $P_{d}$.

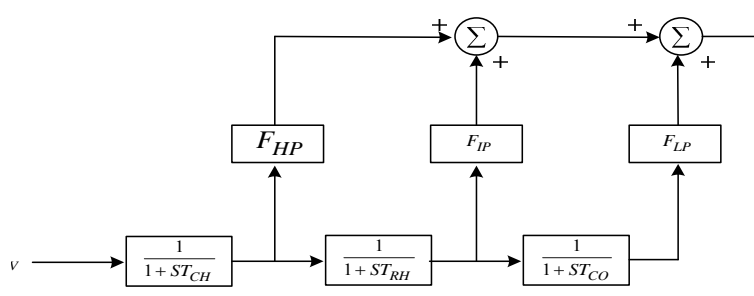

Figure 2. Block Diagram of a single reheat steam turbine.

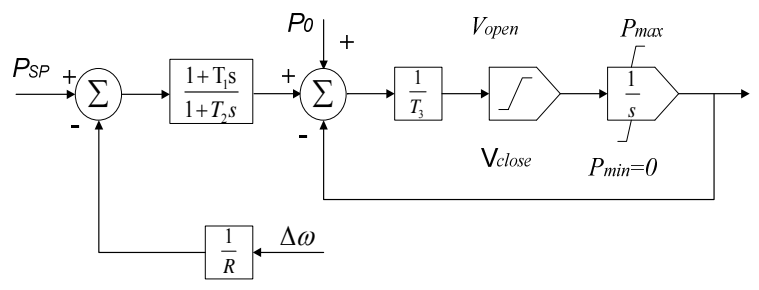

Figure 3. Block diagram of a typical speed governor.

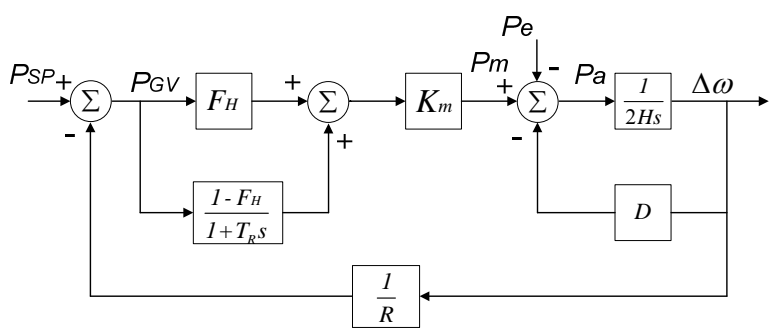

Figure 4. The reduced order SFR (system frequency response) model.
For the situation that load is higher than the active output power in isolated network, we can make $P_{d}=$ $-P_{e}$ corresponding to the stepped load change. It is opposite when load is less than the active output power.

We take the typical parameters of power system as:

$$
\begin{aligned}
& K_{m}=0.95, D=1.0, H=4.0, \\
& T_{R}=8.0, F_{H}=0.3, R=0.05 .
\end{aligned}
$$

We only consider the situation when load is higher than the active output power, and the load unbalances are $10 \%, 20 \%, 30 \%, 40 \%, 50 \%$ power deficiency. The frequency response curve is shown in the Figure 5.

Take the typical parameters of power system that the unbalanced load is given $20 \%$, and the differential coefficients are $0.05,0.06,0.07,0.08,0.09,0.1$. The frequency response curve is shown in the Figure 6. From the figure, we can see that differential coefficient has no influence on initial slope of the frequency curve. The main effects are shown on the recovery time, maximum frequency deviation and the steady state frequency deviation.

\section{Under Frequency Load Shedding Scheme for Isolated Power System}

During the isolated network operation time, the adjust amplitude of generator unit is limited because of the lack of units. So the influence of load disturbance on the isolated network is very obvious [9]. The frequency characteristics of isolated network are very dependent on the

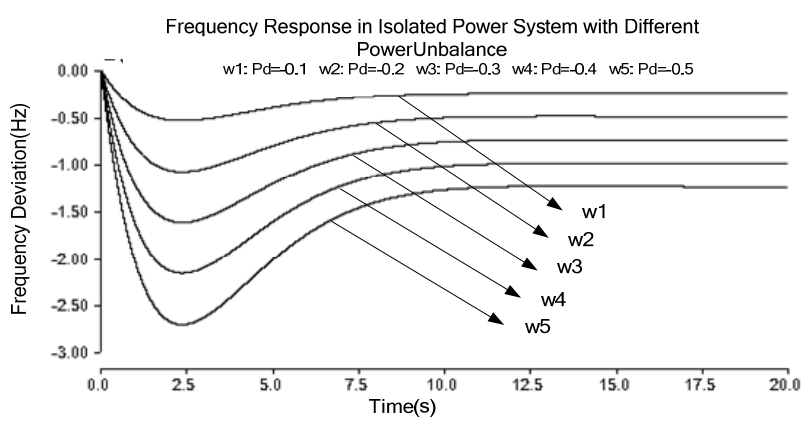

Figure 5. The Frequency Response of Isolated Power System with Different Power Unbalances.

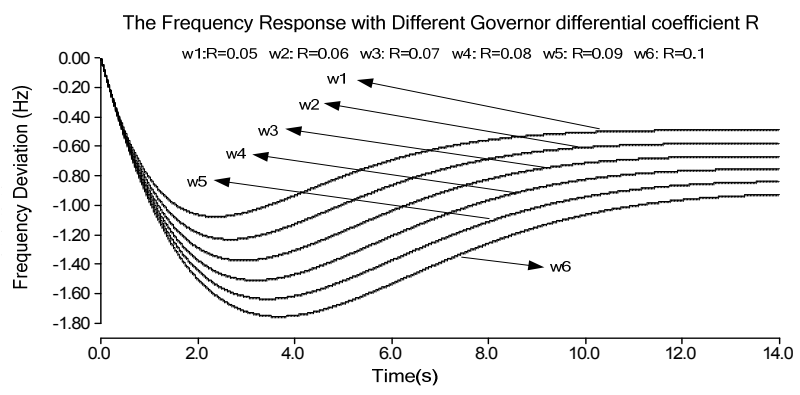

Figure 6. The Frequency Response with Different Governor Differential Coefficient R. 
size of the spinning reserve and frequency regulating characteristics of the generator.

The practical parameters of isolated system operation in a certain power system are taken as an example. The Figure 7 has obviously shown that the generator frequency regulation coefficient reached the maximum value when the frequency is about $49 \mathrm{~Hz}$. Then it decreased with the gradual depletion of spinning reserve. Therefore, when under frequency load shedding scheme is adjusted, it is very necessary to consider the effect of spinning reserve.

The frequency regulation effect coefficient $\mathrm{KS}$ of power system is determined by both of the load and generator frequency regulation characteristics together.

Taking the practical power system as an example, we make assessment of possible isolated system operation on each regional network. Here we use the regional power grid A and B to simulate. We assume that it is the loss of all the $220 \mathrm{kV}$ power supply that causes the isolated system operation. The powers of network A and B are of $50 \%$ and $80 \%$ more than power output. Table 1 is the comparison of the under frequency load shedding scheme between the existing and the optimizing one in the two pieces of power system.

Figure 8(a) shows the existing under frequency load shedding configuration scheme can not effectively restrain frequency from sharply falling. Figure 8(b) shows after adopting optimization load shedding scheme in grid A the lowest value of frequency is $48 \mathrm{~Hz}$ after 5 seconds, and then it returns to $49.6 \mathrm{~Hz}$. Figure 9 shows the frequency curves comparison between the existing and optimizing under frequency load shedding in grid B. The above method is helpful to solve the frequency sharply dropped problem caused by the possible large disturbance occurred when isolated power formed.

The under frequency load shedding is a provincial plan as a whole and is distributed according to cities. For the city field of network in which isolated network operation may appear, the optimization under frequency load shedding scheme is suitable. The deficiency power of the load can be supplied by other pieces of network.

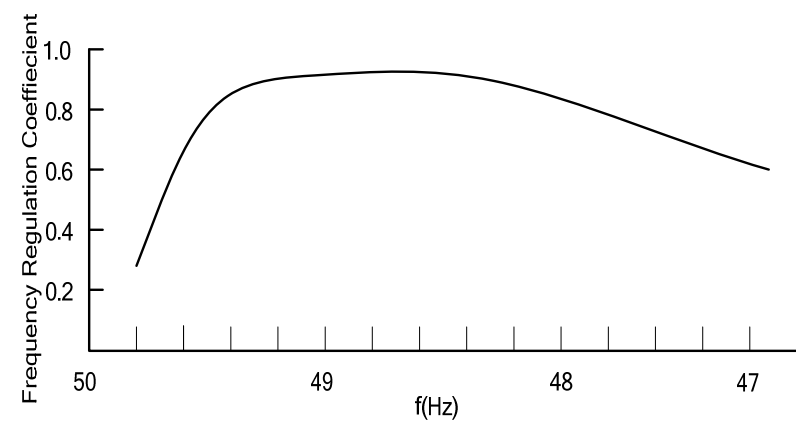

Figure 7. Generator frequency regulation coefficient variation curve with the system frequency variation.
Table 1. Comparison between the existing and optimizing under frequency load shedding scheme in certain pieces of power system.

\begin{tabular}{lccccc}
\hline \multicolumn{1}{c}{ Sheaves } & 1 & 2 & 3 & 4 & 5 \\
\hline Action Frequency(Hz) & 49.0 & 48.8 & 48.6 & 48.4 & 48.2 \\
Delay(s) & 0.2 & 0.2 & 0.2 & 0.2 & 0.2 \\
$\begin{array}{l}\text { Existing load shedding } \\
\text { scheme of power grid A }\end{array}$ & $0 \%$ & $1.5 \%$ & $0 \%$ & $0 \%$ & $10 \%$ \\
Optimization scheme of A & $4.5 \%$ & $5.3 \%$ & $6.1 \%$ & $6.6 \%$ & $7 \%$ \\
Existing scheme of B & $6.8 \%$ & $0 \%$ & $23.6 \%$ & $9.1 \%$ & $0 \%$ \\
Optimization scheme of B & $3.8 \%$ & $4.4 \%$ & $4.9 \%$ & $5.4 \%$ & $5.7 \%$ \\
& & & First & Second & \\
Sheaves & 6 & 7 & special & special & \\
& & & round & round & \\
Action Frequency(Hz) & 48.0 & 47.8 & 49.0 & 49.0 & \\
Delay(s) & 0.2 & 0.2 & 15 & 20 & \\
$\begin{array}{l}\text { Existing load shedding } \\
\text { scheme of power grid A }\end{array}$ & $0 \%$ & $1.5 \%$ & $3 \%$ & $10 \%$ & \\
Optimization scheme of A & $7.2 \%$ & $7.3 \%$ & $3 \%$ & $3 \%$ & \\
$\begin{array}{l}\text { Existing scheme of B } \\
\text { Optimization scheme of B }\end{array}$ & $5.9 \%$ & $6.1 \%$ & $3 \%$ & $3 \%$ & \\
\hline
\end{tabular}

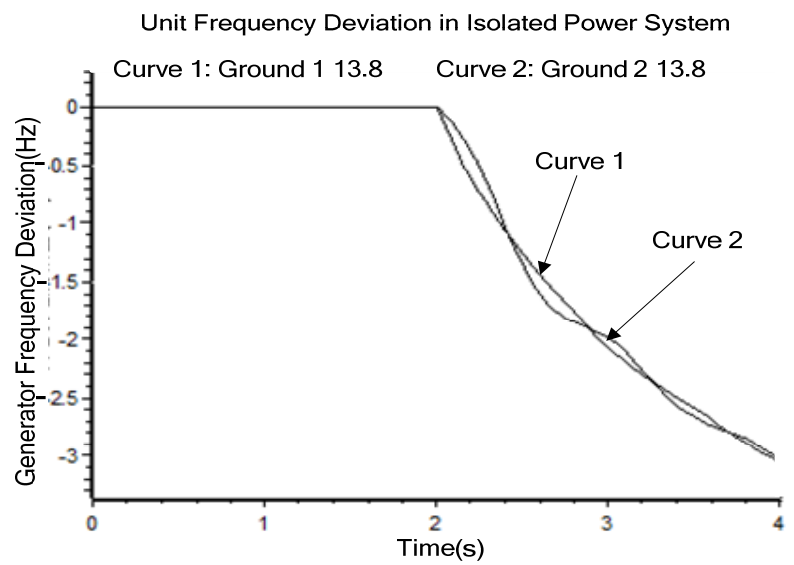

(a) the present scheme

Frequency Curves of the two Units in power plant after low frequency load shedding

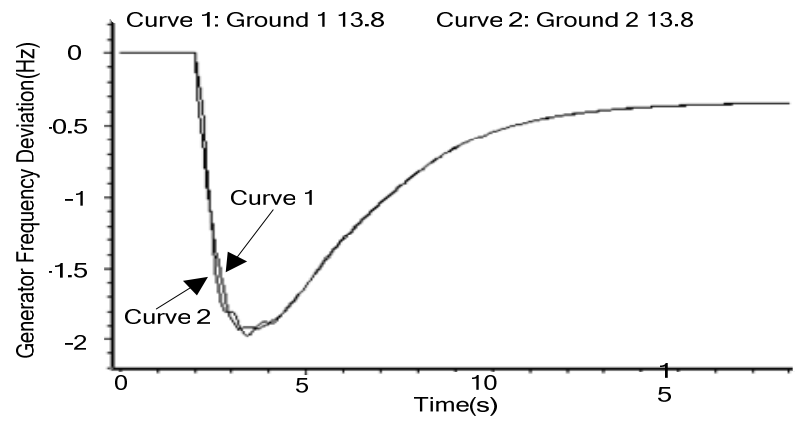

(b) the optimized scheme

Figure 8. Frequency curve comparison of the present and optimized under frequency load shedding schemes in Isolated power system $A$. 


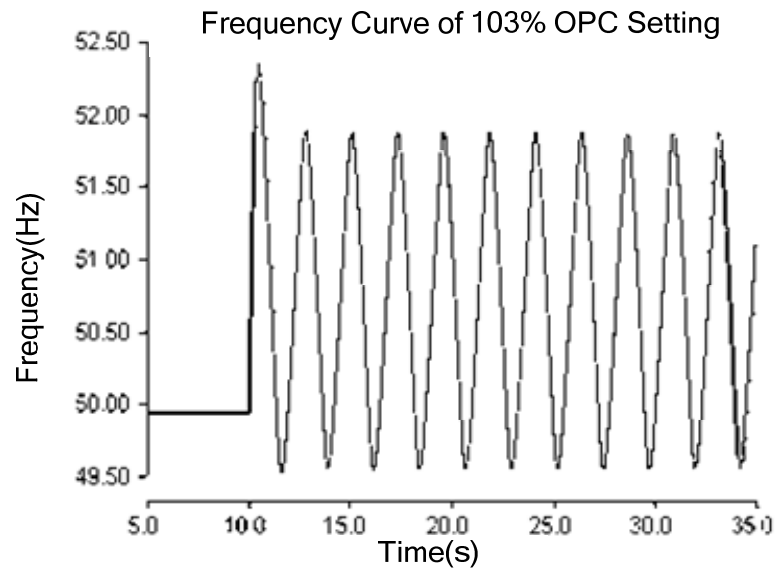

(a) present scheme

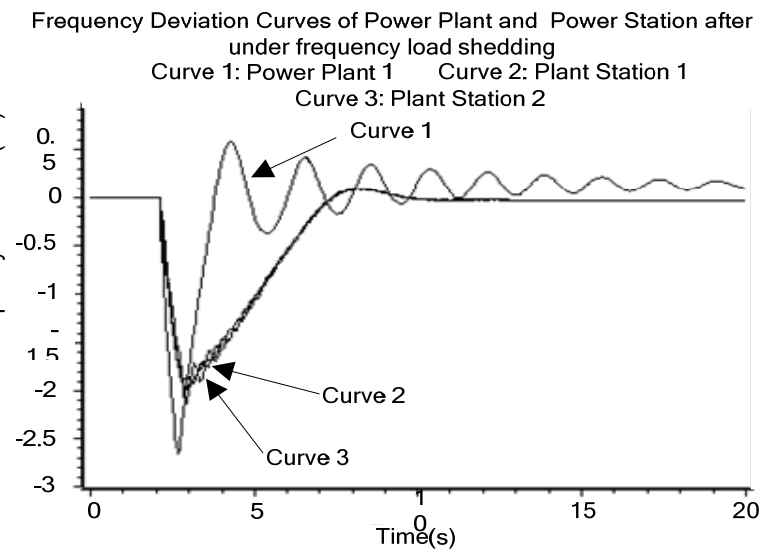

(b) optimized scheme

Figure 9. Frequency curve comparison of the present and optimized schemes load shedding in Isolated Network B.

\section{Control Strategies of Isolated System Operation}

The unbalanced power becomes the main risk for isolated system operation. Generally the unbalanced power of the load is most serious at the early period of isolated power system. If we want the unit to be able to regulate any kind of isolated power system, it needs to adapt to

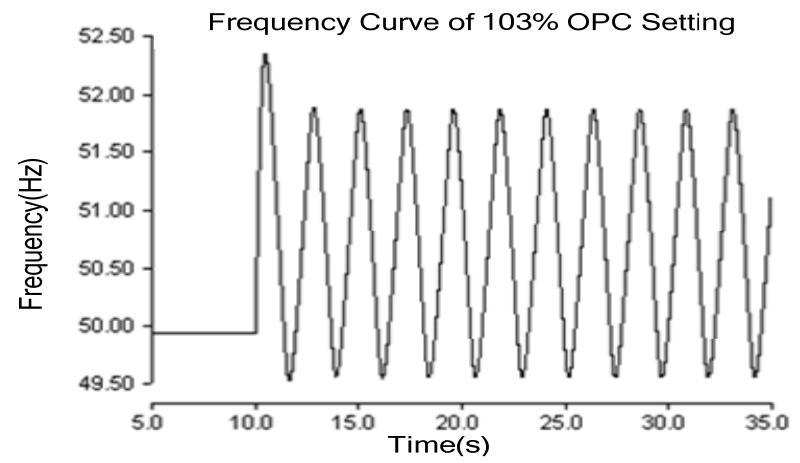

(a) Frequency Curve of $103 \%$ OPC Setting Value the worst conditions. So this paper mainly does the research of the frequency and power response in the case of the relatively large imbalance power disturbance when isolated network forms, and analyzes the corresponding control strategy.

During isolated system operation, primary frequency regulation needs to exit from power control in time, and turns to the valve position control. In order to ensure the safety of the unit, and the stability of isolated system operation, OPC can not quit. For the primary frequency regulation of the unit, the differential coefficient is expected to set lowly. This can make the power response in time after the frequency changes, and prevent the significant change of frequency. Setting the dead band in a low level can optimize the dynamic regulating process of frequency. It is helpful for the stability of frequency to enlarge the top and bottom frequency regulation limitations, and also for reducing the oscillation. Relatively fast response of the servo system can increase quickness of primary frequency regulation of the unit.

There is a frequency deviation after primary frequency regulation. But turbine is not allowed to run with this deviation of frequency for a long time. We can put in the frequency regulation control loop for the speed control during isolated system operation. This is the secondary frequency regulation. Increasing dead band and limitation of primary frequency control appropriately can decrease the number of movements for OPC.

The OPC setting value of the $100 \mathrm{MW}$ unit in one power plant of the power system is respectively taken for 103\% and 106\%. The Figure 10 shows the simulation. Increasing the OPC value appropriately by $2 \%-3 \%$ can reduce frequent opening and closing valve caused by frequency fluctuation.

To sum up, we can make suitable isolated system operation strategy by setting the parameters and control measures of power plant units. The Table 2 takes two certain local power plants in power system as an example, and gives advice about parameter settings of the speed governor.

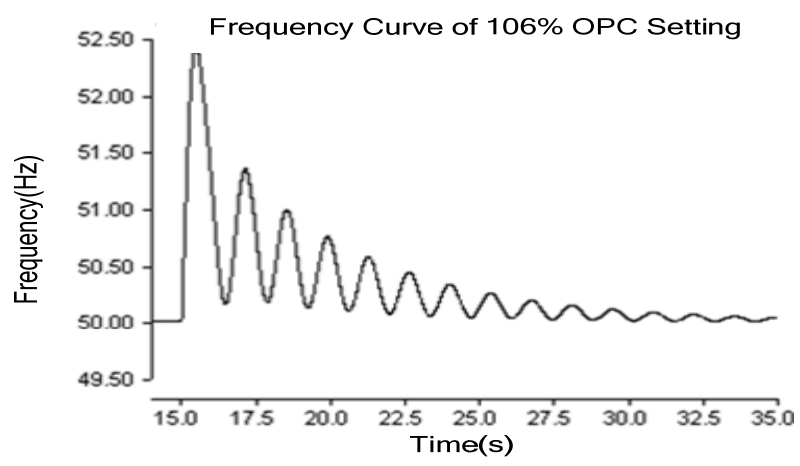

(b) Frequency Curve of 106\% OPC Setting Value

Figure 10. The Frequency Curve of $103 \%$ and $106 \%$ OPC Setting. 
Table 2. The recommendations of parameter value settings of two power plants in power system.

\begin{tabular}{|c|c|c|c|c|}
\hline Power Plant & Types & Parameter Setting & Adjustable Differential Coefficient & Frequency Modulation Limiter \\
\hline \multirow{2}{*}{ A Plant } & \multirow{2}{*}{ Mechanic hydraulic } & scope & $4 \%-5 \%$ & Upper limiter:35\%, no lower limiter \\
\hline & & Proposed value & $4 \%$ & $35 \%$ \\
\hline \multirow{2}{*}{ B Plant } & \multirow{2}{*}{ DEH } & Scope & $4 \%-5 \%$ & Upper limiter:35\%, no lower limiter \\
\hline & & Proposed value & $4 \%$ & $\pm 35 \%$ \\
\hline Power Plant & Types & Parameter Setting & Frequency Modulation Dead Band & OPC Value \\
\hline \multirow{2}{*}{ A Plant } & \multirow{2}{*}{ Mechanic hydraulic } & scope & / & / \\
\hline & & Proposed value & l & l \\
\hline \multirow{2}{*}{ B Plant } & \multirow{2}{*}{ DEH } & Scope & $\leq \pm 0.034 \mathrm{~Hz}$ & $103 \%-108 \%$ \\
\hline & & Proposed value & $\pm 0.017 \mathrm{~Hz}$ & $106 \%$ \\
\hline
\end{tabular}

\section{Conclusions}

This paper analyses the power grid and unit influence factors on the isolated system operation. And the system frequency response model is applied to analyze the specific influences. By simulating and analyzing the stability of regional power system under different load disturbances, regulating system parameters and control logics, parameter settings and control strategies are proposed to improve the ability of isolated system operation. In the view of the frequency characteristic difference between the isolated system operation and networking operation, we have proposed the optimizing scheme of under frequency load shedding which has considered the frequency characteristic differences. This scheme can meet the frequency stability requirement both of networking operation and isolated system operation. Taking the practical power system as an example, we have conducted the real isolated system operation test. This test shows the correctness and effectiveness of the proposed method. The coordinated control strategies improve the ability of power grid and unit to deal with the accident.

\section{REFERENCES}

[1] J. S. Wang, T. T. Deng, J. C. Ran, "Start-up and Operation Mode of Fossil Plant Units in an Isolated (Little) Power Grid," Automation of Electric Power Systems, Vol. 32, No. 21, 2008, pp. 102-106.

[2] H. H. Lin, W. Wang, Y. Y. Wei, "Stable Operation Control Technologies for Islanded Networks in $220 \mathrm{Kv}$ Guilin Power Grid," Southern Power System Technology, Vol. 2, No. 3, 2008, pp. 49-53.

[3] "Causes of the August 14th Blackout in the United States and Canada," US/Canada Power Outage Task Force 2003.

[4] J. Li Jun and P. Luo, "Stability Control Technology for Isolated Network with 300 MW Thermal Power Unit" Guangxi Electric Power, No. 1, 2009, pp. 9-13.

[5] H. Huang, G. H. Xu Guanghu and C. Yu, "Operation Experience of Isolated Network in CSG during Ice Disaster (2008)," Southern Power System Technology, Vol. 2, No. 5, 2008, pp. 6-9.
[6] F. Pilo, G. Celli, S. Mocci, "Improvement to Reliability in Active Networks with Intentional Islanding," IEEE International Conference on Electric Utility Deregulation, Restructuring and Power Technologies, 2004. doi:10.1109/DRPT.2004.1338023

[7] P. M. Anderson. Power System Protection [M]. 2009.

[8] Z. P. Zhou, X. L. Sun, C. P. Fu, K. Liu, "Insular Power System Dynamic Characteristic Examination Research," Power System Technology, Vol. 32, No. 2, 2008, pp. 78-81.

[9] A. M. Li and Z. X. Cai, "Frequency Dynamics Analysis and Load Shedding Assessment Based on the Dynamic Simulation Trajectory of Interconnected Power System," Transactions of China Electrotechnical Society, Vol. 24, No. 9, 2009, pp. 171-177.

[10] J. Wang, X. R. Wang, D. P. Xie, etc, "Investigation of under Frequency Load Shedding on an Isolated System," Power System Protection and Control, Vol. 38, No. 3, 2010, pp. 29-52.

[11] J. Li, "Control Strategy of Single Power Unit with Isolated Operation in Thermal Power Plant," Electric Power, Vol. 41, No. 11, 2008, pp. 51-54.

[12] H. W. Lian, Y. Zhao, "Parameter Optimization for Frequency Modulation of a Unit in Isolated Power Grid Based on Orthogonal Test," Turbine Technology, Vol. 47, No. 2, 2005, pp.108-111.

[13] Z. J. Huang, J. Chao, X. Y. Li and P. Kang, "High Frequency Phenomenon Caused by Fault Occurred in Southern Part of Guiyang Power Grid and Simulation of Overspeed Protection Controller," Power System Technology, Vol. 31, No. 15, 2007, pp. 26-32.

[14] Z. P. Zhou, "First Frequency Modulation Delimitation for Electric System Stabilization Analyse," Henan Electric Power, No. 03, 2009, pp. 40-45.

[15] "IEEE Working Group on Prime Mover and Energy Supply Models for System Dynamic Performance Studies. Dynamic Models for Fossil Fueled Steam Units in Power System Studies," IEEE Transactions on Power System, Vol. 6, No. 2, 1991, pp. 753-761.doi:10.1109/59.76722

[16] F. P. de Mello, Fellow, "Boiler Models for System Dynamic Performance Studies," IEEE Transaction on Power Systems, Vol. 6, No. 1, 1991, pp. 66-74. doi:10.1109/59.131048 